\title{
An Application of Wavelet Analysis to Meat Consumption Cycles
}

\author{
Marija Banović ${ }^{1,2,3^{*}}$, Luis Catela Nunes ${ }^{2}$, Vladan Arsenijevic ${ }^{4}$ \\ ${ }^{1}$ CIISA - Faculty of Veterinary Medicine, Technical University of Lisbon, Lisbon, Portugal; ${ }^{2}$ INOVA—Nova School of Business \\ and Economics, Nova University of Lisbon, Lisbon, Portugal; ${ }^{3} \mathrm{MAPP}$-Aarhus School of Business, Aarhus University, Aarhus, \\ Denmark; ${ }^{4} \mathrm{SIM}$ - Faculty of Sciences, University of Lisbon, Lisbon, Portugal. \\ Email: ${ }^{*}$ admbanovic@fmv.utl.pt
}

Received January $22^{\text {nd }}, 2013$; revised February $22^{\text {nd }}, 2013$; accepted March $4^{\text {th }}, 2013$

\begin{abstract}
The existence or nonexistence of changes in meat consumption cycles is critical to meat industry. If the change is existent, there is a need to understand what motivates the change to identify the most appropriate response. Wavelet analysis is considered here as a promising technique that may lead to a better understanding of characteristic patterns and changes in the meat consumption cycles.
\end{abstract}

Keywords: Meat Consumption Cycles; Wavelet Analysis

\section{Introduction}

Changes in the meat consumption cycles have been the focus of much research over the last decades [1-4]. Even though considerable effort has been put through on studies examining the demand for meats the debate is still going around the factors causing these changes [5-8].

Whatever the explanation may be, the existence or nonexistence of changes in meat consumption cycles is of paramount importance for the meat industry in order to remain competitive in the market. If the change is existent, there is a need to understand what motivates this change in order to identify the most appropriate response. Accordingly, if the explanation behind the change is that consumer preferences have altered and white meat is preferred, industry needs to take action and allocate resources to research, product development and advertising. If the change is nonexistent and changes in meat consumption cycles can be explained by alterations in relative prices then the red meat industry should devote all efforts to produce alternatives that will be able to compete with the white meats on price. Determining whether the changes in the meat consumption cycles have occurred, what motivated them, their duration, and if they repeat over time, is critical for the future industry policies. Inability to detect their occurrence or nonoccurrence could come out costly for the meat industry.

Wavelet analysis has already been successfully applied in a wide variety of disciplines, such as economics, fi-

\footnotetext{
"Corresponding author.
}

nance, physics, and engineering [9-13]. This proficient approach has also been used in agriculture and food quality inspection where it can very well resolve issues of signal processing in various systems used for quality assessment of food products [14]. For example, wavelet analysis has been used to characterize the surface texture of fresh meat giving the predictive models of meat palatability [15].

The typical approach in meat demand studies has been to employ different techniques using only time-domain, giving quite mixed results and complicating any definitive conclusion about the existence or nonexistence of changes in the meat consumption cycles. However, important relations also exist in the frequency domain which cannot be adequately studied using the standard methods. For example, it is possible that consumers react to news about BSE outbreak in the short-run, while in the longrun, the consumption levels are essentially determined by their income. Or, it is possible that the effects of a certain policy may change and evolve over time, therefore affecting the consumption cycles in different ways at different frequencies. The ability to explain the nature and detect dominant patterns in the meat consumption cycles may be one of the most important ways to understand changes in the meat sector, as it also affects other food sectors, both upstream and downstream. Understanding the patterns in the meat consumption cycles may also assist in designing policies affecting the industry and economies where meat production and processing are important economic activities. 
Using the wavelet analysis, this research provides a basis for the identification of characteristic patterns or structures in the meat consumption cycles, which are often masked in the traditional methods. Furthermore, it checks if these patterns exist in other factors (e.g., prices, income), often related to the changes in the meat consumption cycles, and if such relations have evolved over time. For this purpose, several tools are utilized: the wavelet power spectrum, the cross-wavelet spectrum, the wavelet coherence, and the wavelet phase. These techniques show how the use of wavelet analysis may help to unravel time-frequency relationships that would otherwise remain hidden.

This study proceeds as follows. In Section 2, we describe and discuss the wavelet analysis, continuous wavelet transform, wavelet tools, as well as the advantages of wavelet over Fourier transform. Section 3 presents the results of applying wavelet tools to meat consumption cycles and discuss its insights. Section 4 concludes.

\section{Wavelet Analysis}

The wavelet transform is a powerful tool for analyzing economic time-series data characterized by nonlinear dynamics. It allows for a variable-size view of the economic time-series data studying at the same time its time and frequency properties. Thus, the wavelet analysis enables the researcher to separate out a time-series into its constituent multiresolution components [9]. In this way very fine details are detected in a time-series, as well as particular characteristics, patterns, changes, discontinuities and self-similarity that are often some of the most interesting features of a time-series. Furthermore, different relationships between economic variables that can possibly be found at the disaggregate level rather than at an aggregate level may be analyzed. This section intends to provide some intuition and the necessary knowledge for the discussion of the empirical results. For more details about wavelet technique [13,16-18].

\subsection{Continuous Wavelet Transform}

Wavelet analysis examines a time-series by cutting it into small waves of different frequency which are localized in time. In particular, wavelets are obtained by shifting and scaling a specific function $\psi(t)$, called the mother wavelet, and are defined as:

$$
\psi_{\tau, s}(t)=\frac{1}{\sqrt{s}} \psi\left(\frac{t-\tau}{s}\right)
$$

where the parameter $\tau$ determines the wavelet localization in time, and the parameter s determines the scale or width of the wavelet. A low value of the scale parameter $\mathrm{s}$ results in a high frequency wavelet capable of capturing localized short cycles, and vice versa. The large scales (or in terms of frequencies, the low frequencies) correspond to overall information of a time-series, while small scales (high frequencies) correspond to a detailed view. Thus, high frequencies are used to isolate very fine details in a time-series (i.e., changes that occur in a shortrun), while the low frequencies can identify coarse details (i.e., changes that occur in a long-run).

The mother wavelet $\psi(t)$ is simply an oscillating function of time that first grows and then decays and obeys two conditions. The first condition is that the function $\psi(t)$ has a zero mean in the time domain:

$$
\int_{-\infty}^{\infty} \psi(t) \mathrm{d} t=0
$$

The second condition consists of a normalization requirement:

$$
\int_{-\infty}^{\infty}|\psi(t)|^{2} \mathrm{~d} t=1,
$$

and is also known as the unit energy condition. The most commonly used mother wavelet function is the Morlet wavelet given by

$$
\psi^{\mathrm{M}}(t)=\pi^{-\frac{1}{4}} e^{i \omega_{0} t} e^{-\frac{t^{2}}{2}},
$$

where $i$ is the imaginary number and $\omega_{0}$ is the central frequency of the wavelet. In order to study time-varying frequency features, the Morlet wavelet with $\omega_{0}=6$ is an optimal choice, as it gives the best balance between time and frequency domains $[11,17]$. The choice of the mother wavelet will depend on the features of a time-series that will be decomposed. Depending on the application, mother wavelet should be as similar as possible to a time-series (or to its portion under analysis). In our case, and after series of tests with different bases, the Morlet wavelet is found to be the optimal option (choice). The continuous wavelet transform (CWT) of a time-series $x(t)$ with respect to $\psi(t)$ is given by the convolution:

$$
W_{x}(\tau, s)=\int_{-\infty}^{\infty} x(t) \psi_{\tau, s}^{*}(t) \mathrm{d} t,
$$

where the asterisk ${ }^{*}$ denotes the complex conjugate. As with the Fourier counterpart, it is possible to recover the original time-series from the wavelets by using an inverse wavelet transform that integrates over all the time and frequency space.

The CWT of a time-series defined in discrete time $\left\{x_{t} \mid t=1, \cdots, N\right\}$, assuming a uniform unit time step, is obtained as:

$$
W_{x}(\tau, s)=\sum_{t=1}^{N} x_{t} \psi_{\tau, s}^{*}(t) .
$$

Computation of the CWT is usually undertaken over the whole time dimension $\tau$ and over a range of relevant scales $s$. In this manner a fairly good approximation of a given time-series is obtained by using only a few dozen 
coefficients from the coarser scales which is a major advantage in comparison to Fourier transform.

\subsection{Wavelet Tools}

\subsubsection{Wavelet Power Spectrum}

The wavelet power spectrum (WPS) is defined as $\left|W_{x}(\tau, s)\right|^{2}$, and provides a measure of local variance, as it measures the contribution of each scale, or frequency, to the time-series variance, at each point in time. If the WPS is integrated over time, the global wavelet power spectrum (GWPS) is obtained:

$$
G W P S_{x}(s)=\int\left|W_{x}(\tau, s)\right|^{2} \mathrm{~d} \tau,
$$

which can be used for comparison with classical spectral methods that measure the overall contribution of each frequency to the overall time-series variance. Thus, the wavelet power spectrum shows how the variance of a time series is changing over time.

To study the relationships between two time-series $x_{t}$ and $y_{t}$ in both frequency and time domain, one can use the cross-wavelet power (XWT), the wavelet coherence (WCT), and the wavelet phase which generalize the basic univariate wavelet concepts.

\subsubsection{Cross-Wavelet Spectrum}

The cross-wavelet spectrum (XWT) of two time-series $\mathrm{x}_{\mathrm{t}}$ and $y_{t}$ captures the covariance in the time-frequency space and is defined as $\left|W_{x y}(\tau, x)\right|=\left|W_{x}(\tau, s) W_{y}^{*}(\tau, s)\right|$, where $W_{x}$ and $W_{y}$ are the wavelet transforms of $x_{t}$ and $y_{t}$, respectively. The XWT gives a measure of common power between two time-series. While the wavelet power spectrum depicts the variance of a time-series, the crosswavelet transform of two time-series demonstrates covariance between these time-series in the time-frequency space. Thus, XWT shows quantified indication of the similarity of power between two time-series.

\subsubsection{Wavelet Coherence and Phase}

The wavelet coherence (WCT) is obtained from the cross spectrum and provides a measure of the local linear relation between two time-series in the time-frequency plane. It is given by:

$$
R^{2}(\tau, s)=\frac{\left|S\left(s^{-1} W_{x y}(\tau, s)\right)\right|^{2}}{S\left(s^{-1}\left|W_{x}(\tau, s)\right|^{2}\right) S\left(s^{-1}\left|W_{y}(\tau, s)\right|^{2}\right)},
$$

where $S$ is a smoothing operator in both time and scale. The WCT is always between 0 and 1 and may be also seen as an extension of the traditional $R^{2}$ coefficient, as it gives a measure of the strength of the relationship between two time-series in the time-frequency plane. It is equal to 1 when there is a perfect linear relationship between the two time-series at the particular time and scale.
The wavelet phase is a useful tool to characterize the phase relationship between two time-series, i.e., their phase difference. The wavelet phase is defined as:

$$
\phi(\tau, s)=\tan ^{-1} \frac{\mathcal{I}\left(S\left(s^{-1} W_{x y}(\tau, s)\right)\right)}{\% \mathcal{R}\left(S\left(s^{-1} W_{x y}(\tau, s)\right)\right)},
$$

with $\phi_{(x, y)} \in[-\pi, \pi]$, and $I$ and $R$ denote the imaginary and real parts, respectively. The wavelet phase captures lead-lag relationships between two time-series in time and scale. A phase difference of zero suggests that two time-series move together at the specified frequency, which corresponds to positive covariance. In this case, if $\phi_{(x, y)} \in[0, \pi / 2]$ then two time-series move in-phase, but the time-series y leads $x$; while if $\phi_{(x, y)} \in[-\pi / 2,0]$ then $\mathrm{x}$ leads $\mathrm{y}$. On the other hand, a phase difference of $\pi$ (or $-\pi$ ) denotes an anti-phase relationship, which corresponds to negative covariance; where if $\phi_{(x, y)} \in[\pi / 2, \pi]$ then $x$ is leading, and if $\phi_{(x, y)} \in[-\pi / 2,-\pi]$ then $y$ is leading.

\section{Wavelet Analysis and Meat Consumption Cycles}

Annual data for the US per capita consumption of beef, pork, poultry (including chicken and turkey consumption), and fish, as well as beef and pork prices were obtained from the on-line sources of the United States Department of Agriculture (USDA) for the 1909-2008 period for a total of $\mathrm{N}=100$ observations. Prices were deflated using the CPI index (base period 1982-1984=100) from the United States Bureau of Labor Statistics. For per capita GDP the on-line source of the Historical Statistics of the World Economy was used. These series are shown in Figure 1. The wavelet analysis is applied to the growth rates of these series in order to characterize their cycles. Figure 1 shows that the time-series are more likely to exhibit different degrees of temporal stationarity (i.e., non-stationary behavior) which represents no problem for wavelet analysis equipped to deal with characteristics of time-series that more traditional approaches like Fourier analysis cannot handle [16]. Moreover, the length of the time-series ( $\mathrm{N}=100$ observations) is adequate to gather statistically reliable information about occurrence of long cycles.

\subsection{Wavelet Power Spectrum: Identifying Patterns in the Meat Consumption Cycles}

Wavelet analysis allowed us to decompose each of the given time-series as a function of frequency and time. Figure 2 shows the estimated wavelet power spectrum (WPS) for US beef, pork, poultry, and fish consumption, as well as for beef and pork prices and income per capita. The estimated WPS in Figure 2 characterize variance of the time-series across the time-frequency plane. The black 

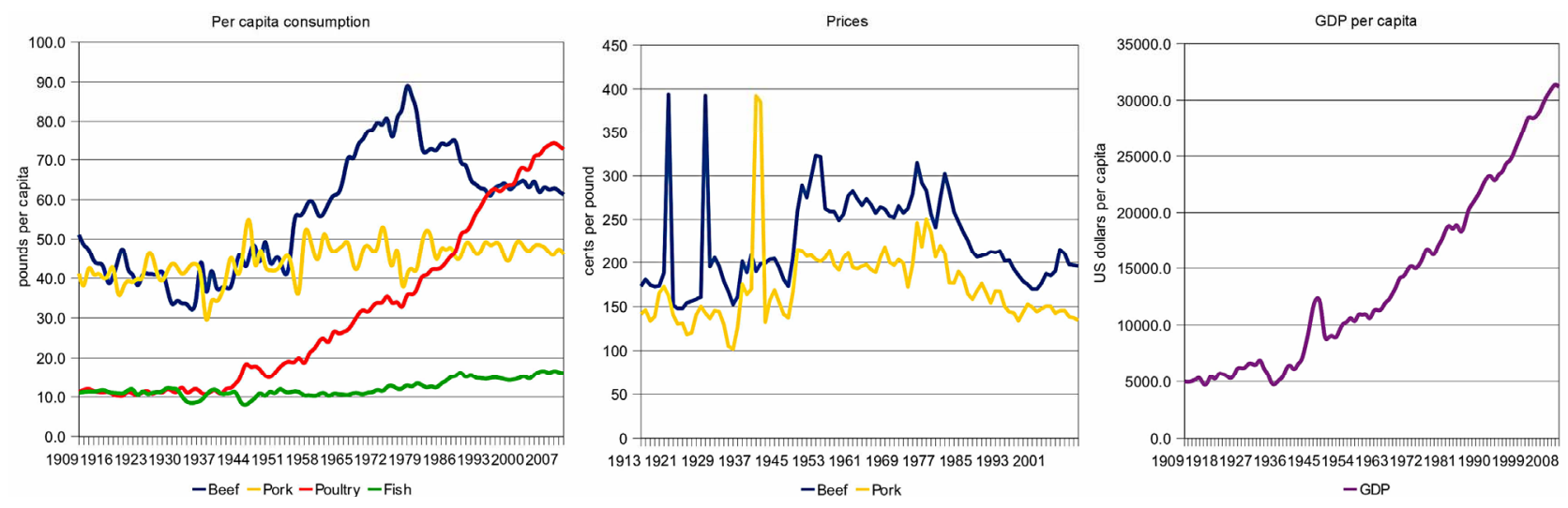

Figure 1. Time-series.

contour designates the $5 \%$ significance level. The regions with high variance (or high power) are presented by red color, while the regions with low variance (or low power) are depicted by blue color.

Figure 2 points to some characteristic patterns of the time-series. The common characteristic of the analyzed time-series is that they exhibit a greater volatility until the late 1950s, which is reflected by higher WPS (i.e., the variance of the time-series). This is quite unlike the second half of the century where volatility decreases, pointing to the beginning of the 1960s as a significant step in time when a structural change occurred.

The analysis of the WPS further reveals two types of common cycles across meats: short ones with a periodicity of 2 - 4 years, which characterize beef, pork, and poultry consumption; and longer cycles with a periodicity of around 8 years for the beef and poultry consumption. Especially interesting are the short cycles during the first half of the century when the time-series are characterized by greater volatility. On the other hand, the longer cycles of beef and poultry disappear after the 1950s.

An important finding is that the pork consumption behaves in a rather unique manner when compared to the other meats. Pork consumption exhibits no evidence of long cycles, only short cycles with 2 - 4-year periodicity during the 1970s. Two types of mechanism are responsible for this distinct behavior of pork consumption. First, the more stable and repetitive short cycles in pork consumption point to the insensitivity of pork consumption to changes in own prices and incomes (see next two subsections). Second, the fact that short cycles continue throughout the 1970s is particularly interesting, and this is probably influenced by changes in production practices in the US pork industry during the 1970s. In that decade the US pork industry improved breeding and husbandry practices and began trimming outside fat on retail cuts, which lowered the fat content of pork [8]. This might have led consumers to perceive pork as healthier (containing less fat, calories and cholesterol) reflecting more stability in pork consumption.

The estimated WPS of beef and pork prices differ. The pork prices display shorter cycles with 2 - 6 years periodicity until the $1950 \mathrm{~s}$, while beef prices show rather long cycles with 2 - 10-year periodicity around the 1940s. Income has a major peak close to the 8 year periodicity, coinciding with the long cycles and a minor peak at the 2 - 4-year band corresponding to the shorter cycles in meat consumption found earlier. These cycles correspond to the business cycle frequencies reported in the literature (see e.g. [10]). As seen from Figures 2(e)-(g) these cycles appear only up to the mid 1950s, which is in line with the Great Moderation period found for the US economy after this time.

\subsection{Cross-Wavelet Spectrum: Understanding Common Features in the Meat Consumption Cycles}

The cross-wavelet power spectrum (XWT) estimate shows the common features of any two time-series. Figure 3 demonstrates the pattern similarity in the time-series. The black contour designates the $5 \%$ significance level. The power similarity between two time-series is depicted by color system ranging from blue-low covariance to redhigh covariance. The relative phase relationship is shown as arrows with in-phase (positive covariance) pointing right, and anti-phase (negative covariance) pointing left.

The results from Figure 3 confirm that there is a significant covariance between the different time-series at the shorter and longer cycles identified during the first half of the century. In particular, the XWT of beef and poultry consumption suggests a large interdependence between these two time-series at 2 - 4-year cycles from the early 1920 s until the late $1950 \mathrm{~s}$, as well as in the $6-$ 12 -year cycles from the early 1930s until the late 1950s. However, the XWT of pork and poultry consumption exhibits highly significant areas of interdependence from the 1920s to the late 1970s. Interestingly, poultry and fish consumption show a large area of common power but in 

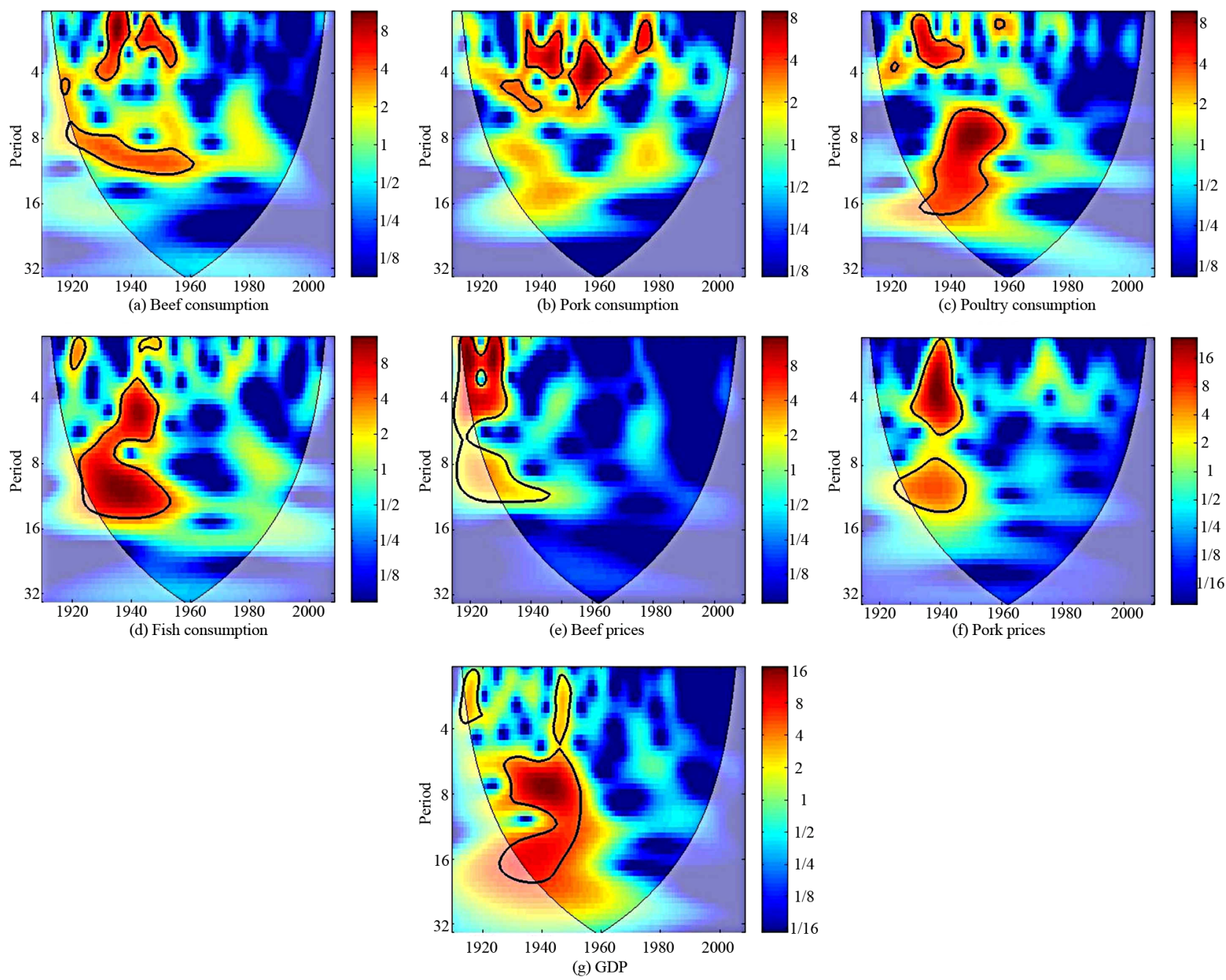

Figure 2. Estimated wavelet power spectra (WPS) of time-series growth rates. The $x$-axis represents time in years, while the y-axis shows period in years. The black contour designates the $5 \%$ significance level. The regions with high variance (or high power) are presented by red color, while the regions with low variance (or low power) are depicted by blue color.

the 2 - 16-year band during the late 1920s until the 1960s. Pork and fish consumption exhibit quite a similar pattern.

Figure 3(j) presents the relationship between income and different types of meat and fish consumption where the late 1950s and the beginning of 1960s has again been confirmed as a turning-point in meat consumption timeseries, when significant changes occurred. The estimated XWT shows higher sensitivity of meat consumption to income changes until the late 1950s, shown by joint power both at low and high scales. A similar pattern has been observed for the relationship between beef consumption and own prices. Nevertheless, the pork consumption exhibits less sensitivity to own price, after the late 1950s.

A similar pattern occurs in the association between income and fish consumption. Since the relationship between income and meat consumption, as well as income and fish consumption, is mainly in positive covariance across all scales, it can be confirmed increased sensitivity of meat and fish consumption to changes in income. This is also evident even outside the cone of influence, pointing to important links between income and meat consumption even after the decade of 2000s.

Since the XWT exposes regions with common features (high common power) between two time-series, but does not explain their local cross-correlation, i.e., their strength of relationship, we further analyze wavelet coherence and phase.

\subsection{Wavelet Coherence and Phase: Unraveling Strength of Factors Influencing the Meat Consumption Cycles}

The cross-wavelet coherence (WCT) checks for a significant correlation between different time-series. Figure 4 displays the WCT estimates of the cross-correlation between the different time-series as a function of cycle 


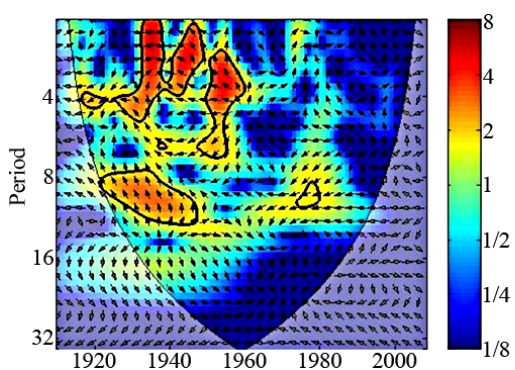

(a) Beef and Pork consumption
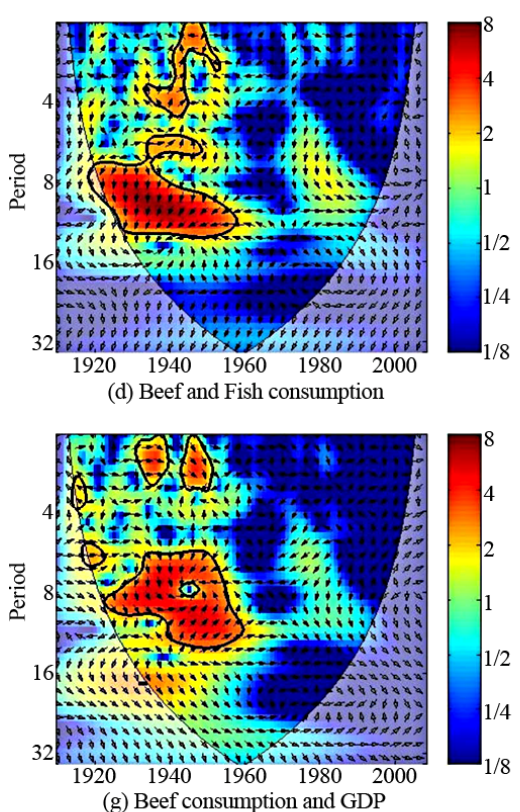

(g) Beef consumption and GDP
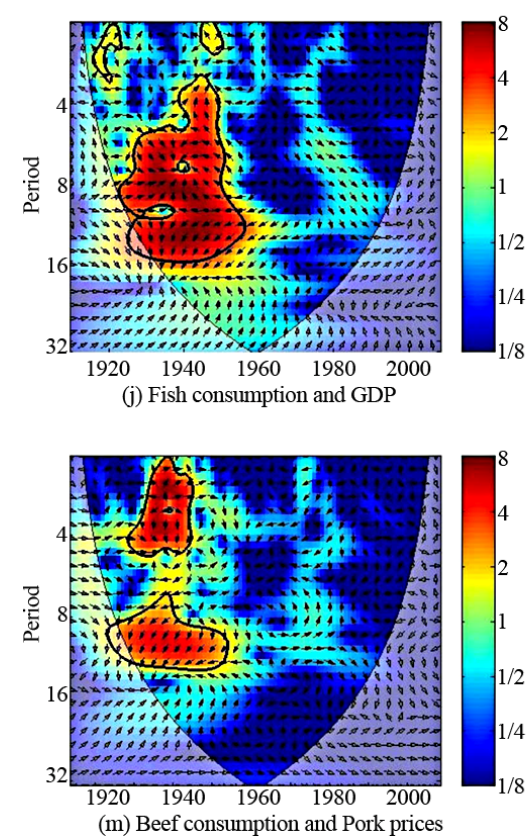

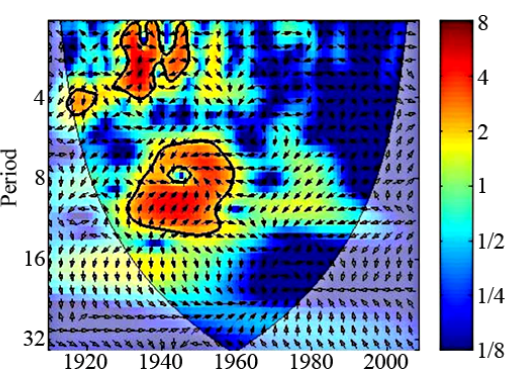

(b) Beef and Poultry consumption

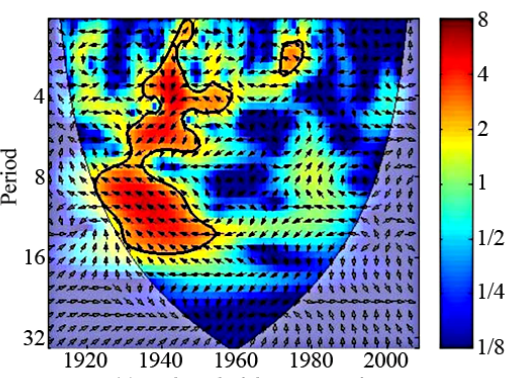

(e) Pork and Fish consumption

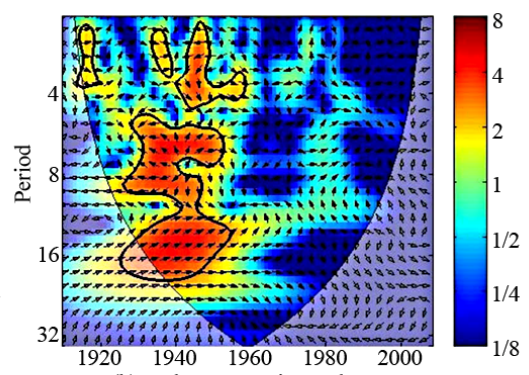

(h) Pork consumption and GDP

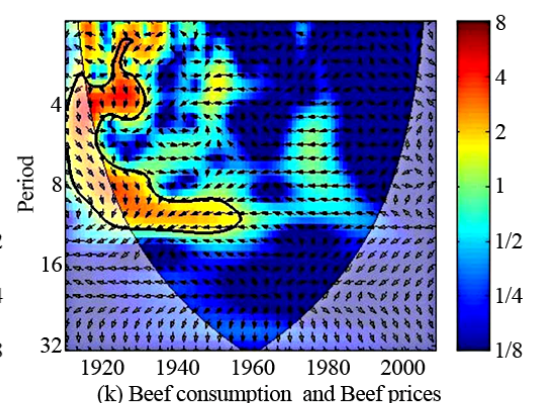

(k) Beef consumption and Beef prices

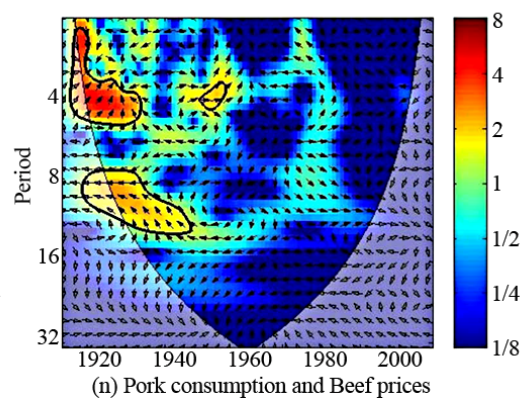

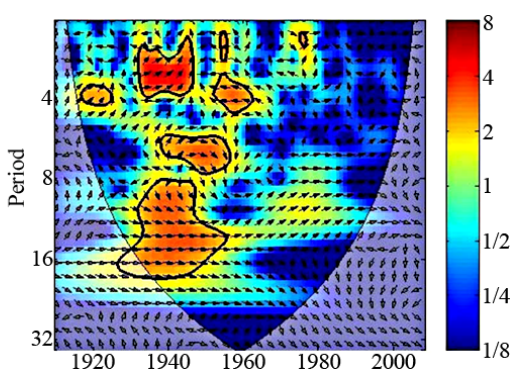

(c) Pork and Poultry consumption

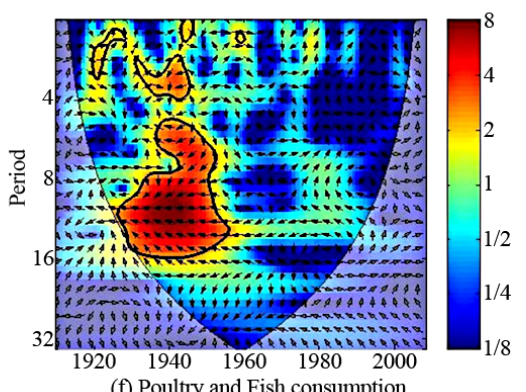

(f) Poultry and Fish consumption

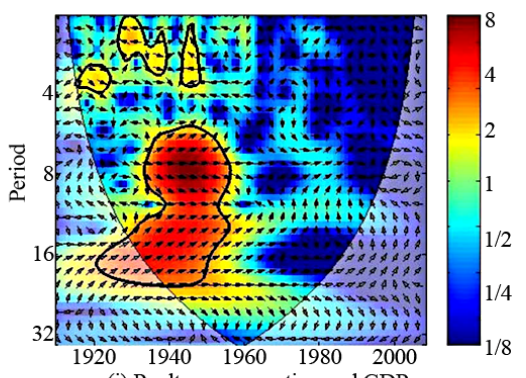

(i) Poultry consumption and GDP

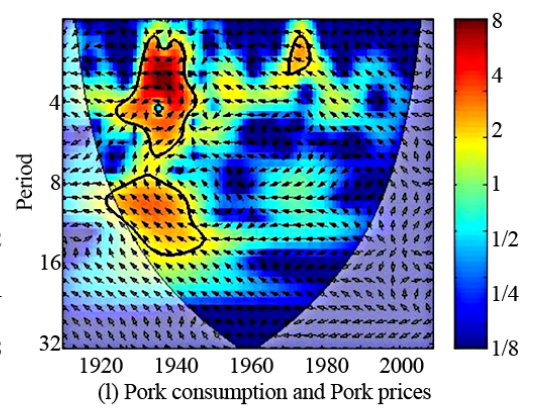

(l) Pork consumption and Pork prices

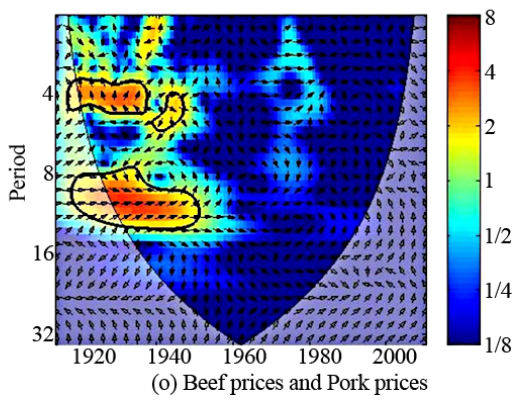

Figure 3. Estimated cross-wavelet transforms (XWT) of the time-series growth rates. The $x$-axis represents time in years, while the $y$-axis shows period in years. The black contour designates the $\mathbf{5 \%}$ significance level. The power similarity between two time-series is depicted by color system ranging from blue-low covariance to red-high covariance. The relative phase relationship is shown as arrows with in-phase (positive covariance) pointing right, and anti-phase (negative covariance) pointing left. 


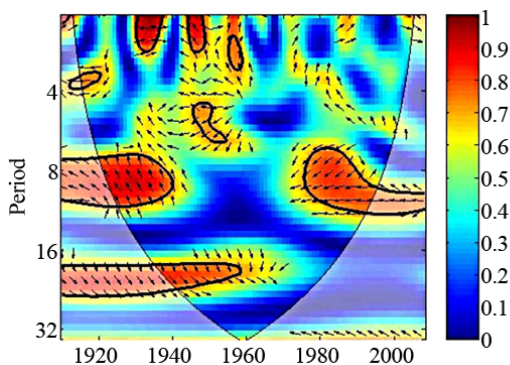

(a) Beef and Pork consumption

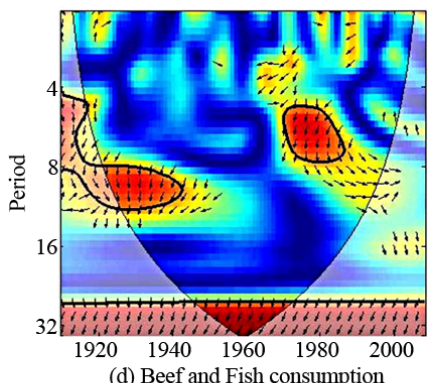

(d) Beef and Fish consumption
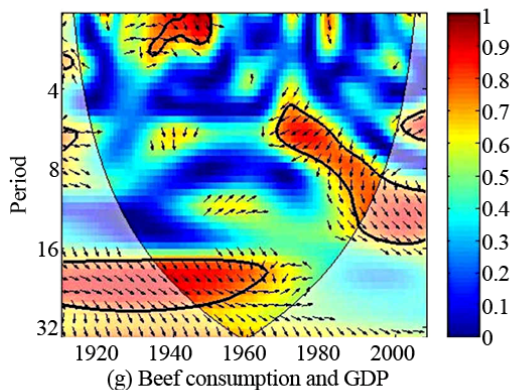

(g) Beef consumption and GDP

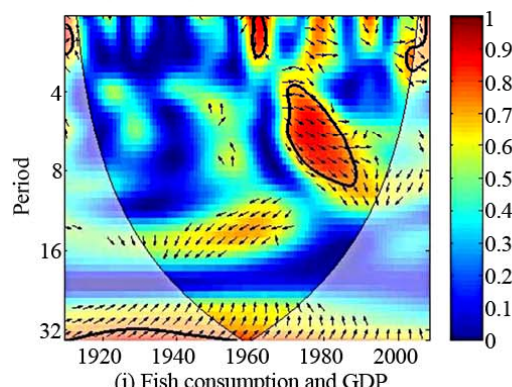

(j) Fish consumption and GDP

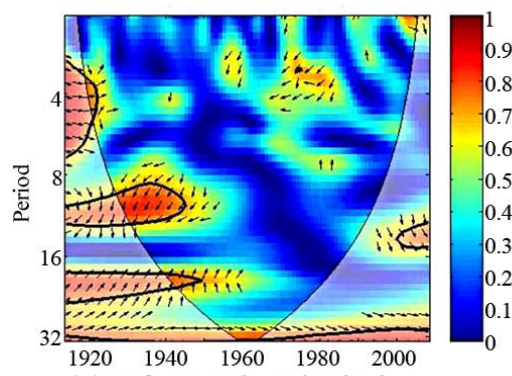

(m) Beef consumption and Pork prices

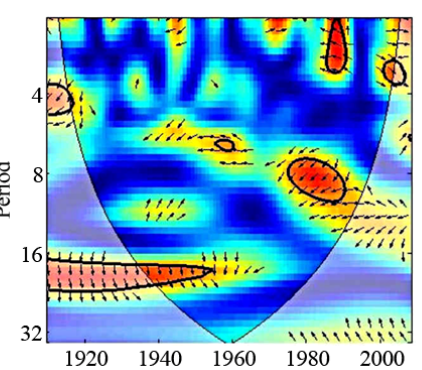

(b) Beef and Poultry consumption

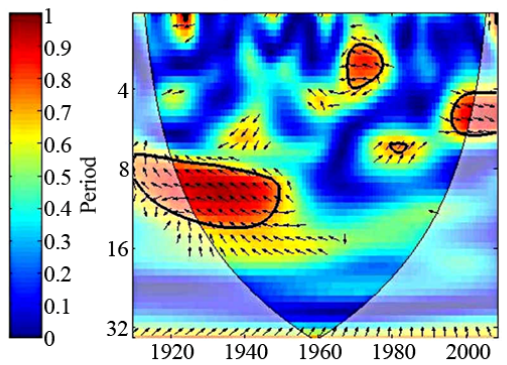

(e) Pork and Fish consumption

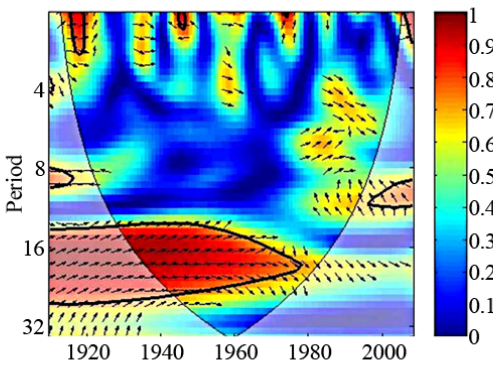

(h) Pork consumption and GDP

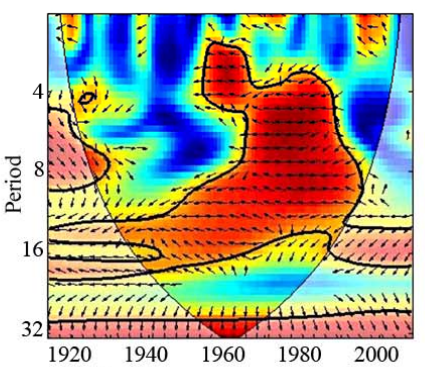

(k) Beef consumption and Beef prices

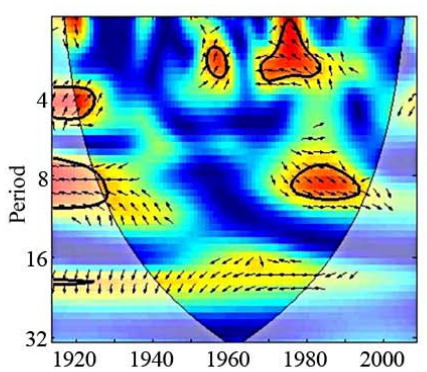

(n) Pork consumption and Beef prices

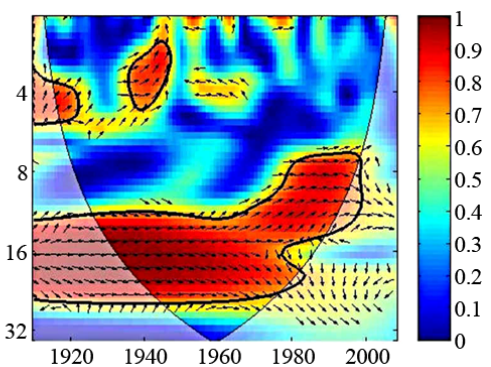

(c) Pork and Poultry consumption

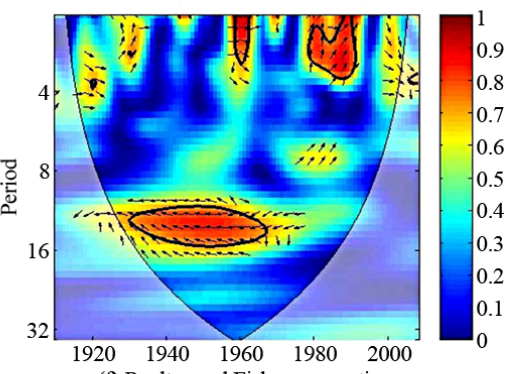

(f) Poultry and Fish consumption

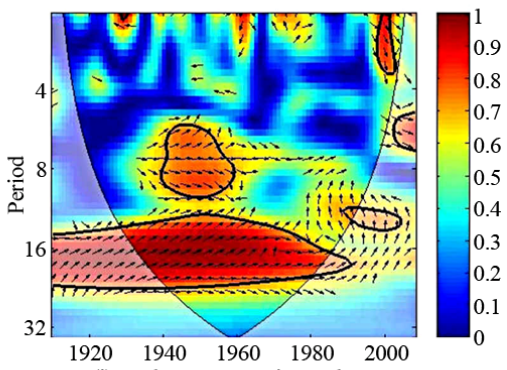

(i) Poultry consumption and GDP

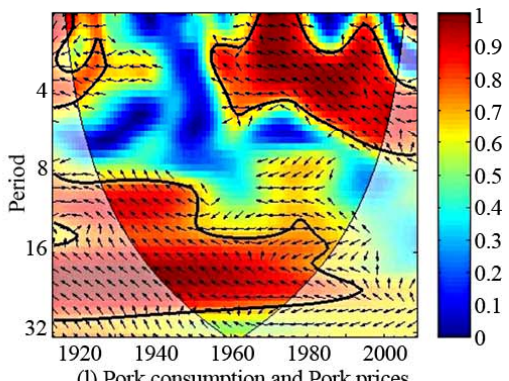

(l) Pork consumption and Pork prices

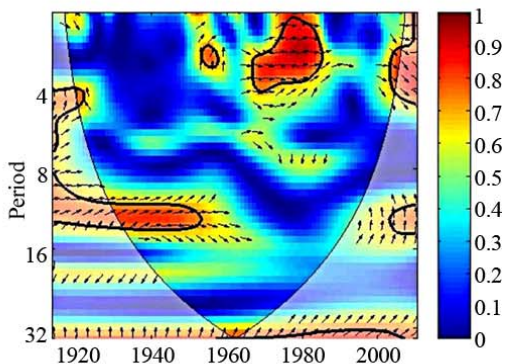

(o) Beef prices and Pork prices

Figure 4. Estimated wavelet coherences (WCT) of time-series growth rates. The $x$-axis represents time in years, while the $y$-axis shows period in years. The black contour designates the $5 \%$ significance level. The strength of relationship between two time-series is explained by a color system ranging from blue-low coherency to red-high coherency. The mutual interaction of time-series is shown by arrows. Arrows pointing to the right mean that the time-series are in-phase, while arrows pointing to the left mean that the time-series are in anti-phase. 
periodicity and time, showing their relationship strength and mutual interaction. The black contour designates the $5 \%$ significance level. The strength of relationship between two time-series is explained by a color system ranging from blue-low coherency to red-high coherency. The mutual interaction (or locally phase locked behavior) among time-series is shown by arrows. Arrows pointing to the right mean that the time-series are in-phase, while arrows pointing to the left mean that the time-series are in anti-phase. When two time-series are in-phase arrows pointing right and up mean that first time-series lags behind second time-series, while to the right and down first one leads second one. On the other hand, when two time-series are in anti-phase arrows pointing left and up show that first time-series leads second time-series, and left and down indicate that first one lags behind second one.

Figure 4 exposes several ranges of significant coherence (dependence) between the different time-series. The correlation between beef and poultry consumption is very strong and significant from the beginning of the century until the late $1950 \mathrm{~s}$ in the 16 - 20-year band, during the 1980s in the 8 year band and in the 1990s around 3-year band. For the longer periods and until the 1980s poultry is led by beef, but in the 1990s poultry starts replacing beef. This change in the way meat consumption is distributed across beef and poultry signalize the occurrence of shifts in consumer preferences. This finding coincides with the increased consumers' awareness with health, safety, and nutritional issues during the 1980s and 1990s, when US consumers started to see beef as less desirable than poultry in terms of fat, cholesterol, calorie content, artificial ingredients, convenience characteristics, store display, and price (too expensive) $[2,4,19]$.

Figure 4(m) reveals a strong dependence of beef consumption to changes in pork price, especially around 10year band. These time-series mutually interact in a way that beef consumption is actually influenced by pork prices. Moreover, Figure 4(n) demonstrates dependence of beef prices to changes in pork consumption in the 8 year band, when pork consumption during the 1980s and 1990s leads beef prices. Figure 4(o) also shows that beef and pork prices are highly interrelated and negatively correlated.

A strong interrelationship between beef and fish consumption is also observed in Figure 4(d), which is especially evident for the large cycle periodicity of 30 years. It seems that fish in the long run might evolve from being an unimportant commodity to a good that will be an important future protein opponent to meat. In fact, Wohlgenant [19] pointed out that during the mid 1970s, the relationship between beef and fish changed from one of substitutability to one of complementarity. Nevertheless, our results demonstrate that this relationship has been prolonged to the end of 2000s.

The relationship between pork and poultry consumption, Figure 4(c), is very synchronous (in-phase) around the 16-year band and for all time periods, demonstrating similar movement of their consumption cycles. The sectors outside the cone of influence and above the 5\% significance level are not a reliable indicator of correlation, however, the significant area of is so large that it is very unlikely to be simply by chance, revealing again substitution between these two meats. Figure 4(e) shows that the coherence of pork and fish consumption is very significant for a periodicity of 8 years at the beginning of the century and until the $1950 \mathrm{~s}$, as well as in the $3-4$ year periodicity during the 1970s. These two series are negatively correlated (in anti-phase), where fish consumption lags behind pork consumption. However, the behavior of these two time-series changes completely during the late 1990s and 2000s, exhibiting a synchronized pattern. This suggests that unlike the period until the 1980 s, when fish and pork consumption alternate, substituting one another, during the 1990s and 2000s they tend to fluctuate in a similar manner. These are significant results that again point to the exceptional behavior of pork consumption cycles, which may be related to the fact that in the 1990s pork contained more lean and less fat due to the new production practices, in which the US pork industry actually capitalized by promoting pork as a light and nutritious alternative to poultry-"Pork: The Other White Meat" [8]. Comparing the results for the relationship between meat and fish consumption and income (Figures 3 and 4), we see that the larger areas stand out as being significant, showing a positive (inphase) relationship between the aforementioned timeseries, suggesting that meat and fish consumption accompany changes in income. The beef, pork, and poultry consumption cycles with periodicities around 20 years are strongly correlated with income cycles.

In Figure 4 the regions of higher coherence at lower to medium scales may point to what is perhaps the most interesting element of change regarding the relationship between income and meat consumption (beef and poultry). While income and beef consumption, as well as income and poultry consumption, show high mutual dependence at medium to high scales and for most of the period, the relationship between income and pork consumption is more concentrated at higher scales. The increased sensitivity of beef and poultry consumption to changes in income, and decreased sensitivity of pork consumption to income again points to uniqueness of pork consumption cycles. The US pork industry has managed to tackle the challenge of meeting the consumer demands regarding pork that seems to be an entirely different product in the eyes of the consumers [8].

Unlike the association between income and meat con- 
sumption, the relationship between income and fish consumption accounts for much smaller regions of coherence mostly at medium scales. This occurs during the decade of the 1970s until the late 1980s, when the relationship between fish consumption and income displays a strong cross-correlation that points to fish becoming an important protein source at par with meat.

Our results show that meat consumption time-series can significantly alter both in time and frequency and that wavelet tools are good indicators of these alterations. The finding that the beginning of 1960s represents an important turning point in the evolution of US meat consumption cycles, and that this significant structural change was not completed until the decade of the 2000s, is a rather important contribution to the research on meat consumption cycles. Moreover, the wavelet analysis undertaken here demonstrates not only changes of the meat consumption cycles in time, but also in frequency. The findings of the different meat consumption patterns is also particularly interesting, especially pork consumption cycles that exhibit more stability at the shorter cycles, showing no evidence of long cycles, and thus pointing to the fact that the pork consumption is less sensitive to external shocks.

\section{Conclusions}

This paper has used wavelet analysis to understand characteristic features, patterns and changes in the meat consumption cycles for the United States data. The realworld data, such as meat consumption time-series, often have complex dynamics that dominate their behavior. Wavelet analysis has the potential to deal with nonstationary data, while most econometric methods assume stationarity, which may or may not be apparent in economic data. Furthermore, wavelets ability to separate out the dynamics in a time-series over a different time horizons, can reveal interesting insights into cycles at different time scales. Besides common analysis in the time domain that can be done using other techniques, wavelet analysis can also capture the hidden information on consumption cycles in the frequency domain, where the evolution of the wavelet transform from one scale to another is easy to follow and reconstruct, isolating very fine details or changes that occur in a short-run (using high frequencies), and very large details or changes that occur in a long-run (using low frequencies). By applying the wavelet approach, we have shown its potential in examining meat consumption cycles, as well as interactions between different time-series, representing them in a time-frequency plane where features and patterns (we could not otherwise see) can be observed in an analytical way.

The US meat industry has been a major force in US agriculture and is currently undergoing significant change.
In order to be able to respond to consumer demand and adapt production patterns and practices it is important to capture any systematic pattern that eventually occurs in the meat consumption cycles, especially at coarse scales which determine global trends, as they influence global environment. Our analysis has demonstrated significant structural change in meat consumption cycles initiated in the 1960s and not completed until the decade of the 2000s, patterns of two types of prevailing cycles: longer with 8-year periodicity and shorter with 2 - 4-year periodicity characterized by greater volatility. Moreover, we have shown that pork consumption exhibit more stability at the shorter cycles, showing no evidence of long cycles. Facing changes in the meat sector the identification and understanding of the specificities of these global trends could help the meat industry to make this information useful and effective. Moreover, found patterns occurring in the short time at fine scales that do not affect overall run are also of concern as they give the information on the shocks that occurred and might occur in the short-run. Not only do identified patterns describe meat consumption cycles, but they also encompass the overall dynamics and the short- and long-run effects of income and prices along years that might lead to unexpected trends and (rather) local instability. In other words, wavelet analysis besides identifying patterns in the meat consumption cycles can help us to conclude if these patterns exist in other factors, like prices and income, and if such relations evolve over time. Through simple observation of the wavelet estimates it has found a mutual interaction and similar patterns between beef consumption and pork price, especially with 10 -year periodicity. On the other hand, it has been demonstrated that pork consumption leads beef prices with the 8-year periodicity. The capacity of demystifying the nature and detecting the dominant features and patterns in the meat consumption cycles is significant for understanding of changes in the meat sector. This may assist in designing policies affecting the other food sectors, as well as industry and economies where meat production and processing are important economic activities.

The insights from wavelet analysis on meat consumption cycles may come handy for further improvements of the existent models currently used in the analyses of meat demand. Generally, this will involve complementing these models with the frequency dimension which can point to the underlying patterns in meat consumption which cannot be seen otherwise. What would be especially pertinent, and what is generally missing in demand models, as well as in our analysis, are variables describing product attributes and consumer characteristics that may influence the meat consumption cycles. The identification of these additional variables, other than prices and income, could further contribute to a better understanding of 
changes in the meat consumption cycles and factors behind these alterations. This challenging task could further enable more appropriate industry responses to shifts in consumer preferences. Thereby, if these latter are existent and point toward higher dietary and health consciousness then meat industry could allocate appropriate resources to research, product development and advertising. On the other hand, if the shifts in consumer preferences are nonexistent and changes in meat consumption can be explained only by alterations in prices and income then the industry could concentrate to producing more competitive alternatives.

\section{Acknowledgements}

M. Banović is supported by Fundação para a Ciência e a Tecnologia through grant SFRH/BPD/63067/2009.

\section{REFERENCES}

[1] J. Chavas, "Structural Change in the Demand for Meat," American Journal of Agricultural Economics, Vol. 65, No. 1, 1983, pp. 148-153. doi:10.2307/1240351

[2] G. Moschini and D. Meilke, "Parameter Stability and the US Demand for Beef," Western Journal of Agricultural Economics, Vol. 9, No. 2, 1984, pp. 271-282.

[3] B. Goodwin, "Multivariate Gradual Switching Systems and the Stability of US Meat Demands: A Bayesian Analysis," Structural Change and Economic Dynamics, Vol. 3 No. 1, 1992, pp. 155-166. doi:10.1016/0954-349X(92)90032-2

[4] E. W. Anderson and S. M. Shugan, "Repositioning for Changing Preferences-The Case of Beef versus Poultry," Journal of Consumer Research, Vol. 18, No. 2, 1991, pp. 219-232. doi:10.1086/209254

[5] J. Eales and L. Unnevehr, "Demand for Beef and Chicken Products: Separability and Structural Change," American Journal of Agricultural Economics, Vol. 70, No. 3, 1988, pp. 521-532. doi: $10.2307 / 1241490$

[6] R. Robenstein and W. Thurman, "Health Risk and the Demand for Red Meat: Evidence from Futures Markets," Review of Agricultural Economics, Vol. 18, No. 4, 1996, pp. 629-641.

[7] G. Tonsor and N. Olynk, "Impacts of Animal Well-Being and Welfare Media on Meat Demand," Journal of Agricultural Economics, Vol. 62, No. 1, 2010, pp. 59-72. doi:10.1111/j.1477-9552.2010.00266.x

[8] A. Resurreccion, "Sensory Aspects of Consumer Choices for Meat and Meat Products," Meat Science, Vol. 66, No 1, 2001, pp. 11-20. doi:10.1016/S0309-1740(03)00021-4

[9] P. Crowley, "A Guide to Wavelets for Economists," Journal of Economic Surveys, Vol. 21, No. 2, 2007, pp. $207-$ 267. doi:10.1111/j.1467-6419.2006.00502.x

[10] A. Rua and L. Nunes, "International Comovement of Stock Market Returns: A Wavelet Analysis," Journal of Empirical Finance, Vol. 16, No. 4, 2001, pp. 632-639. doi:10.1016/j.jempfin.2009.02.002

[11] A. Grinsted, J. Moore and S. Jevrejeva, "Application of the Cross Wavelet Transform and Wavelet Coherence to Geophysical Time Series," Nonlinear Processes in Geophysics, Vol. 11, No. 5/6, 2004, pp. 561-566. doi:10.5194/npg-11-561-2004

[12] V. Arsenijevic, "A New Insight into the Classification of Type Ia Supernovae," Monthly Notices of the Royal Astronomical Society (MNRAS), Vol. 414, No. 2, 2011, pp. 1617-1624. doi:10.1111/j.1365-2966.2011.18493.x

[13] V. Arsenijevic, S. Fabbro, A. M. Mourão and A. J. Rica da Silva, "Diversity of Supernovae Ia Determined Using Equivalent Widths of Si II 4000," Astronomy \& Astrophysics, Vol. 492, No. 2, 2008, pp. 535-544. doi:10.1051/0004-6361:200810675

[14] C. B. Singh, R. Choudhary, D. S. Jayas and J. Paliwal, "Wavelet Analysis of Signals in Agriculture and Food Quality Inspection," Food and Bioprocess Technology, Vol. 3, No. 1, 2010, pp. 2-12. doi:10.1007/s11947-008-0093-7

[15] P. Jackman, D.-W. Sun and P. Allen, "Comparison of Various Wavelet Texture Features to Predict Beef Palatability," Meat Science, Vol. 83, No. 1, 2009, pp. 82-87. doi:10.1016/j.meatsci.2009.04.003

[16] I. Daubechies, "Ten Lectures on Wavelets," 9th Edition, Society for Industrial and Applied Mathemathics, Philadelphia, 1992. doi:10.1137/1.9781611970104

[17] C. Torrence and G. Compo, "A Practical Guide to Wavelet Analysis," Bulletin of the American Meterological Society, Vol. 79, No. 1, 1998, pp. 61-78. doi:10.1175/1520-0477(1998)079<0061:APGTWA>2.0. $\mathrm{CO} ; 2$

[18] C. Torrence and P. Webster, "The Annual Cycle of Persistence in the El Niño-Southern Oscillation," Quarterly Journal of the Royal Meteorological Society, Vol. 124, No. 550, 1998, pp. 1985-2004. doi:10.1002/qj.49712455010

[19] M. Wohlgenant, "Estimating Cross Elasticities of Demand for Beef," Western Journal of Agricultural Economics, Vol. 10, No. 2, 1985, pp. 322-329. 